Fixed Point Theory, 22(2021), No. 1, 231-240

DOI: $10.24193 /$ fpt-ro.2021.1.16

http://www.math.ubbcluj.ro/ nodeacj/sfptcj.html

\title{
EXISTENCE OF THREE WEAK SOLUTIONS FOR A CLASS OF DISCRETE PROBLEMS DRIVEN BY $p$-LAPLACIAN OPERATOR
}

\author{
MAURIZIO IMBESI*, RAHMATOLLAH LASHKARIPOUR** AND ZAHRA AHMADI** \\ *Department of Mathematical and Computer Sciences, Physical and Earth Sciences, \\ University of Messina, Viale F. Stagno d'Alcontres 31, 98166 Messina, Italy \\ E-mail: imbesim@unime.it \\ ** Department of Mathematics, Faculty of Mathematics, \\ University of Sistan and Baluchestan, Zahedan, Iran \\ E-mails: z.ahmadiz@yahoo.com, lashkari@hamoon.usb.ac.ir
}

\begin{abstract}
In this paper, by using a theorem based on variational method which was recently proved by Ricceri, we establish the existence of three weak solutions for a class of $p$-Laplacian discrete problems. Remarks and examples are provided to illustrate our result.

Key Words and Phrases: Discrete boundary value problem, $p$-Laplacian discrete equations, three solutions, critical point theory.
\end{abstract}

2020 Mathematics Subject Classification: 46E39, 58E05, 47H10.

\section{REFERENCES}

[1] F.M. Atici, A. Cabada, Existence and uniqueness results for discrete second-order periodic boundary value problems, Comput. Math. Appl., 45(2003), 1417-1427.

[2] F.M. Atici, G.Sh. Guseinov, Positive periodic solutions for nonlinear difference equations with periodic coefficients, J. Math. Anal. Appl., 232(1999), 166-182.

[3] G. Bonanno, P. Candito, Nonlinear difference equations investigated via critical point methods, Nonlinear Anal. TMA, 70(2009), 3180-3186.

[4] G. Bonanno, P. Jebelean, C. Şerban, Three solutions for discrete anisotropic periodic and Neumann problems, Dynamic Sys. Appl., 22(2013), 183-196.

[5] A. Cabada, A. Iannizzotto, S. Tersian, Multiple solutions for discrete boundary value problem, J. Math. Anal. Appl., 356(2009), 418-428.

[6] J. Chu, D. Jiang, Eigenvalues and discrete boundary value problems for the one-dimensional p-Laplacian, J. Math. Anal. Appl., 305(2005), 452-465.

[7] M. Galewski, S. Heidarkhani, A. Salari, Multiplicity results for discrete anisotropic equations, Discrete Contin. Dyn. Syst. Ser. B, 23(2018), 203-218.

[8] J.R. Graef, S. Heidarkhani, L. Kong, A. Salari, Three weak solutions to a degenerate quasilinear elliptic system, Le Matematiche, 74(2019), no. 1, 191-210.

[9] S. Heidarkhani, G.A. Afrouzi, J. Henderson, S. Moradi, G. Caristi, Variational approaches to p-Laplacian discrete problems of Kirchhoff type, J. Differ. Equ. Appl., 23(2017), 917-938.

[10] S. Heidarkhani, G.A. Afrouzi, M. Imbesi, S. Moradi, Existence of three weak solutions for a perturbed anisotropic discrete Dirichlet problem, Appl. Anal., 98(2019), no. 3, 561-580. 
[11] S. Heidarkhani, M. Imbesi, Multiple solutions for partial discrete Dirichlet problems depending on a real parameter, J. Diff. Equat. Appl., 21(2015), no. 2, 96-110.

[12] S. Heidarkhani, M. Imbesi, Nontrivial solutions for partial discrete Dirichlet problems via a local minimum theorem for functionals, J. Nonlin. Funct. Anal., 2019(2019), art. ID 42, 1-14.

[13] J. Henderson, H.B. Thompson, Existence of multiple solutions for second order discrete boundary value problems, Comput. Math. Appl., 43(2002), 1239-1248.

[14] M. Imbesi, G. Molica Bisci, Discrete elliptic Dirichlet problems and nonlinear algebraic systems, Mediterr. J. Math., 13(2016), no. 1, 263-278.

[15] L. Jiang, Z. Zhou, Three solutions to Dirichlet boundary value problems for p-Laplacian difference equations, Adv. Diff. Equ., 2008(2008), 1-10.

[16] W.G. Kelly, A.C. Peterson, Difference Equations: An Introduction with Applications, Academic Press, San Diego, New York, Basel, 1991.

[17] G. Molica Bisci, A. Pansera, Three weak solutions for nonlocal fractional equations, Adv. Nonlinear Stud., 14(2014), 619-629.

[18] G. Molica Bisci, D. Repovš, Existence of solutions for p-Laplacian discrete equations, Applied Math. Comput., 242(2014), 454-461.

[19] B. Ricceri, A further refinement of a three critical points theorem, Nonlinear Anal. TMA, 74(2011), 7446-7454.

[20] D.B. Wang, W. Guan, Three positive solutions of boundary value problems for p-Laplacian difference equations, Comput. Math. Appl., 55(2008), 1943-1949.

Received: January 10, 2019; Accepted: November 21, 2019. 